\title{
Rotation-Function Space Groups
}

\section{By S. Narasinga RaO, Jyh-Hwang JiH* and Jean Ann Hartsuck}

\section{Laboratory of Protein Studies, Oklahoma Medical Research Foundation and Department of Biochemistry and Molecular Biology, University of Oklahoma, at Oklahoma City Health Sciences Center, Oklahoma City, Oklahoma 73104, USA}

(Received 19 November 1979; accepted 30 April 1980)

\begin{abstract}
Space groups of the 100 possible rotation functions which do not involve cubic crystallographic symmetry have been derived. All 100 belong to one of 16 basic space groups, but many possess additional translational symmetry. Asymmetric units for Eulerian coordinates and for $\theta_{+}, \theta_{-}$coordinates are tabulated.
\end{abstract}

\section{Introduction}

Since its conception in 1962, the rotation function (Rossmann \& Blow, 1962) has been used in many laboratories to determine the orientation of a known molecule in a different crystal or to define noncrystallographic rotational symmetry within a crystal. Tollin, Main \& Rossmann (1966) developed a method for calculating the symmetry of the rotation function. We have now applied that method to all possible combinations of non-cubic space groups. One hundred such pairs exist, but several combinations yield the same symmetry elements so that there are only 16 possible space groups for all the rotation functions from non-cubic space groups. Lattman (1972) suggested a modification of the coordinate system for the rotation function. We also tabulate asymmetric units for the rotation space groups in the Lattman $\theta_{+}, \theta_{-}$coordinate system.

\section{Derivation of the rotation space groups}

As explained by Tollin et al. (1966), the symmetry of the rotation function can be deduced by exhaustive combination of all equivalent positions in the proper rotation groups of the two Patterson functions which

\footnotetext{
* Present address: Department of Chemistry, University of Pennsylvania, Philadelphia, PA 19174, USA. An earlier version of this work constituted a portion of the dissertation research of Jyh-Hwang Jih in partial fulfilment of the requirements for the PhD degree in Biochemistry and Molecular Biology at the University of Oklahoma, at Oklahoma City Health Sciences Center.
}

are being compared. The three Eulerian angles $\theta_{1}, \theta_{2}$ and $\theta_{3}$ are used to form a three-dimensional coordinate system. The unit cell is $2 \pi$ along each axis since $\theta$ and $2 \pi+\theta$ are the same rotation. Each equivalent rotation is considered an equivalent position in the rotation space. Fortunately, the rotation space groups thus formed are members of the crystallographic space group set described in International Tables for X-ray Crystallography (1969). Table 1 lists the symmetry elements in Eulerian angles for the proper rotation groups of the nine non-cubic Laue groups. Each group includes the operation $\pi+\theta_{1},-\theta_{2}, \pi+\theta_{3}$, which is an identity operation in the Eulerian system.

Since the Eulerian rotation matrix is not Hermitian, reversing the order of the Patterson functions in the rotation function does not produce the same rotation-

Table 1. Symmetry elements $S_{i}$ and ${ }_{j} S$ for all proper rotation groups except cubic ones

This table is compiled from Tables 1 and 2 of Tollin et al. (1966).

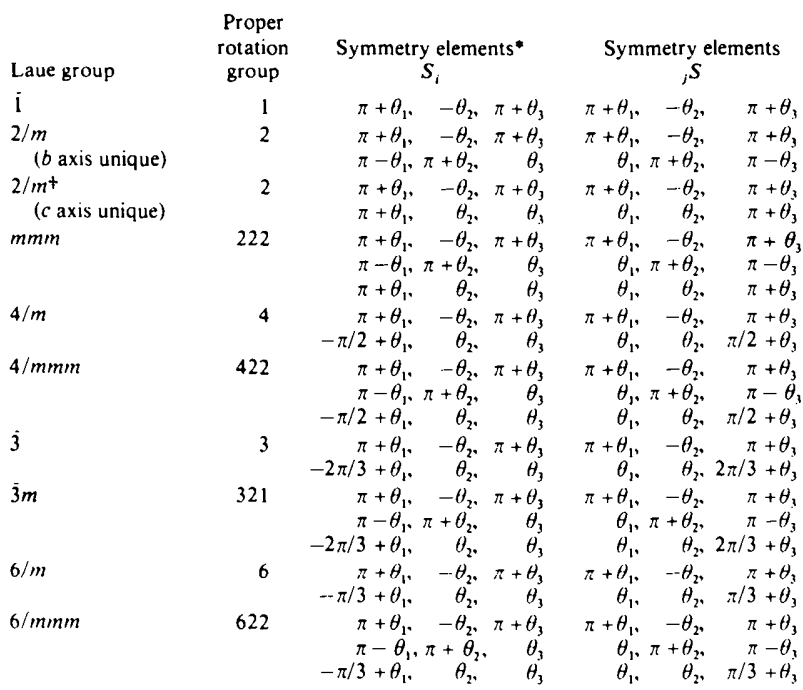

* Proper rotation-group symmetry elements $S_{\text {, and }} S$ are applied successively to generate a rotation-function symmetry element $S_{i} . S_{i}$ is an element in the Patterson map which is rotated.

+ The two monoclinic settings ( $b$ axis or $c$ axis unique) are not separate Laue groups but are treated individually here since they produce different results in the rotation function.

(C) 1980 International Union of Crystallography 
function equivalent positions. Consequently, we treat reverse order in the rotation function as an independent case even though the two arrangements do yield related equivalent positions (Tollin et al., 1966).

As an example, suppose the Patterson map to be rotated $\left(P_{1}\right)$ has Laue symmetry $P 6 / m$ and the other Patterson map $\left(P_{2}\right)$ has symmetry $P 2 / m$ with the twofold rotation axis parallel to $b$. From Table 1 , the Laue group symmetry elements $S_{i}$ and ${ }_{j} S$ are:

$$
\begin{aligned}
& S_{1}\left(\theta_{1}, \theta_{2}, \theta_{3}\right) \rightarrow\left(\pi+\theta_{1},-\theta_{2}, \pi+\theta_{3}\right) ; \\
& S_{2}\left(\theta_{1}, \theta_{2}, \theta_{3}\right) \rightarrow\left(-\pi / 3+\theta_{1}, \theta_{2}, \theta_{3}\right) ; \\
& { }_{1} S\left(\theta_{1}, \theta_{2}, \theta_{3}\right) \rightarrow\left(\pi+\theta_{1},-\theta_{2}, \pi+\theta_{3}\right) ; \\
& { }_{2} S\left(\theta_{1}, \theta_{2}, \theta_{3}\right) \rightarrow\left(\theta_{1}, \pi+\theta_{2}, \pi-\theta_{3}\right) .
\end{aligned}
$$

An exhaustive combination of these $S_{i}$ and ${ }_{j} S$ results in 24 unique rotation-function symmetry elements ${ }_{j} S_{i}$. Multiple application of the sixfold rotation element is required to generate all possible equivalent positions. Another example of this type of treatment has already been given (Tollin et al., 1966). For each of the $24, S_{i}$ below, one of the combinations of $S_{i}$ 's and ${ }_{j} S$ 's which yield each ${ }_{j} S_{i}$ is shown in parentheses. Other combinations exist which yield the same equivalent position ${ }_{j} S_{i}$ :

$\begin{array}{rrrl}\theta_{1}, & \theta_{2}, & \theta_{3} & \\ \theta_{1}, & -\theta_{2}, & \pi+\theta_{3} & \left(S_{2} \cdot S_{2} \cdot S_{2} \cdot S_{1}\right) \\ \theta_{1}, & \pi+\theta_{2}, & \pi-\theta_{3} & (2) \\ \theta_{1}, & \pi-\theta_{2}, & -\theta_{3} & \left(S_{2} \cdot S_{2} \cdot S_{2} \cdot S_{1} \cdot{ }_{2} S\right) \\ \pi / 3+\theta_{1}, & \theta_{2}, & \theta_{3} & \left(S_{2} \cdot S_{2} \cdot S_{2} \cdot S_{2} \cdot S_{2}\right) \\ \pi / 3+\theta_{1}, & -\theta_{2}, & \pi+\theta_{3} & \left(S_{2} \cdot S_{2} \cdot S_{1}\right) \\ \pi / 3+\theta_{1}, & \pi+\theta_{2}, & \pi-\theta_{3} & \left(S_{2} \cdot S_{2} \cdot S_{2} \cdot S_{2} \cdot S_{2} \cdot{ }_{2} S\right) \\ \pi / 3+\theta_{1}, & \pi-\theta_{2}, & -\theta_{3} & \left(S_{2} \cdot S_{2} \cdot S_{1} \cdot{ }_{2} S\right) \\ 2 \pi / 3+\theta_{1}, & \theta_{2}, & \theta_{3} & \left(S_{2} \cdot S_{2} \cdot S_{2} \cdot S_{2}\right) \\ 2 \pi / 3+\theta_{1}, & -\theta_{2}, & \pi+\theta_{3} & \left(S_{2} \cdot S_{1}\right) \\ 2 \pi / 3+\theta_{1}, & \pi+\theta_{2}, & \pi-\theta_{3} & \left(S_{2} \cdot S_{2} \cdot S_{2} \cdot S_{2} \cdot 2\right) \\ 2 \pi / 3+\theta_{1}, & \pi-\theta_{2}, & -\theta_{3} & \left(S_{2} \cdot S_{1} \cdot S_{2}\right) \\ \pi+\theta_{1}, & \theta_{2}, & \theta_{3} & \left(S_{2} \cdot S_{2} \cdot S_{2}\right) \\ \pi+\theta_{1}, & -\theta_{2}, & \pi+\theta_{3} & \left(S_{1}\right) \\ \pi+\theta_{1}, & \pi+\theta_{2}, & \pi-\theta_{3} & \left(S_{2} \cdot S_{2} \cdot S_{2} \cdot S_{2} S\right) \\ \pi+\theta_{1}, & \pi-\theta_{2}, & -\theta_{3} & \left(S_{1} \cdot S_{2}\right) \\ 4 \pi / 3+\theta_{1}, & \theta_{2}, & \theta_{3} & \left(S_{2} \cdot S_{2}\right) \\ 4 \pi / 3+\theta_{1}, & -\theta_{2}, & \pi+\theta_{3} & \left(S_{2} \cdot S_{2} \cdot S_{2} \cdot S_{2} \cdot S_{2} \cdot S_{1}\right) \\ 4 \pi / 3+\theta_{1}, & \pi+\theta_{2}, & \pi-\theta_{3} & \left(S_{2} \cdot S_{2} \cdot 2\right) \\ 4 \pi / 3+\theta_{1}, & \pi-\theta_{2}, & -\theta_{3} & \left(S_{2} \cdot S_{2} \cdot S_{2} \cdot S_{2} \cdot S_{2} \cdot S_{1} \cdot 2 S\right) \\ 5 \pi / 3+\theta_{1}, & \theta_{2}, & \theta_{3} & \left(S_{2}\right) \\ 5 \pi / 3+\theta_{1}, & -\theta_{2}, & \pi+\theta_{3} & \left(S_{2} \cdot S_{2} \cdot S_{2} \cdot S_{2} \cdot S_{1}\right) \\ 5 \pi / 3+\theta_{1}, & \pi+\theta_{2}, & \pi-\theta_{3} & \left(S_{2} \cdot{ }_{2} S\right) \\ 5 \pi / 3+\theta_{1}, & \pi-\theta_{2}, & -\theta_{3} & \left(S_{2} \cdot S_{2} \cdot S_{2} \cdot S_{2} \cdot S_{1} \cdot{ }_{2} S\right) .\end{array}$

This rotation-function space group of 24 equivalent positions is made up of a basic group of four rotations plus five related groups which are identical except for consecutive translation by $\pi / 3$ along the $\theta_{1}$ axis. In the basic set the space group $P 2 c b$ can be recognized by examining the symmetry properties shown in brackets below:

$$
\begin{aligned}
& \theta_{1}, \quad \theta_{2}, \quad \theta_{3} \\
& \theta_{1}, \quad-\theta_{2}, \pi+\theta_{3}(c \text { glide perpendicular to } \mathbf{b}) \\
& \theta_{1}, \pi+\theta_{2}, \pi-\theta_{3}(b \text { glide perpendicular to } \mathbf{c}) \\
& \theta_{1}, \pi-\theta_{2}, \quad-\theta_{3}(2 \text {-fold axis along } \mathbf{a}) .
\end{aligned}
$$

For every rotation space group, there are several consistent choices of the range of the asymmetric unit. But when the rotation function is calculated as a function of $\theta_{+}$and $\theta_{-}$in sections of constant $\theta_{2}$ (Lattman, 1972), it is advantageous to minimize the range of $\theta_{2}$. For this reason, we chose the asymmetric unit for this example to be:

$$
\begin{aligned}
& 0 \leq \theta_{1}<\pi / 3 \\
& 0 \leq \theta_{2} \leq \pi / 2 \\
& 0 \leq \theta_{3}<2 \pi .
\end{aligned}
$$

We wrote a Fortran computer program which listed all possible combinations of a group of input symmetry elements. From these lists the equivalent positions for each rotation-function space group were deduced.

Our numbering of the possible rotation-function space groups is listed in Table 2. Once the number of a specific rotation-function space group is known from Table 2, its characteristics can be easily found in Table 3 , in which all the possible rotation-function space groups, except those involving cubic Laue groups, are listed. In the 100 unique combinations of Laue groups, there are only 16 basic rotation-function space groups, whose equivalent positions are listed in Table 4. All rotation-function space groups are either one of the 16 listed in Table 4 or one of the 16 basic groups plus

\begin{tabular}{|c|c|c|c|c|c|c|c|c|c|c|}
\hline & $\mathrm{i}$ & $\begin{array}{c}2 / m \\
b \text { axis } \\
\text { unique }\end{array}$ & $\begin{array}{c}2 / m \\
c \text { axis } \\
\text { unique }\end{array}$ & $m m m$ & $4 / m$ & $4 / \mathrm{mmm}$ & 3 & $3 m$ & $6 / m$ & $6 / m m n$ \\
\hline i & 1 & 11 & 21 & 31 & 41 & 51 & 61 & 71 & 81 & 91 \\
\hline $\begin{array}{l}2 / m \\
\quad b \text { axis } \\
\text { unique }\end{array}$ & 2 & 12 & 22 & 32 & 42 & 52 & 62 & 72 & 82 & 92 \\
\hline $\begin{array}{l}2 / m \\
\quad c \text { axis } \\
\text { unique }\end{array}$ & 3 & 13 & 23 & 33 & 43 & 53 & 63 & 73 & 83 & 93 \\
\hline $\mathrm{mmm}$ & 4 & 14 & 24 & 34 & 44 & 54 & 64 & 74 & 84 & 94 \\
\hline $4 / m$ & 5 & 15 & 25 & 35 & 45 & 55 & 65 & 75 & 85 & 95 \\
\hline $4 / \mathrm{mmm}$ & 6 & 16 & 26 & 36 & 46 & 56 & 66 & 76 & 86 & 96 \\
\hline 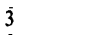 & 7 & 17 & 27 & 37 & 47 & 57 & 67 & 77 & 87 & 97 \\
\hline $3 m$ & 8 & 18 & 28 & 38 & 48 & 58 & 68 & 78 & 88 & 98 \\
\hline $6 / m$ & 9 & 19 & 29 & 39 & 49 & 59 & 69 & 79 & 89 & 99 \\
\hline $6 / \mathrm{mmm}$ & 10 & 20 & 30 & 40 & 50 & 60 & 70 & 80 & 90 & 100 \\
\hline
\end{tabular}
translation along the $\theta_{1}$ and/or the $\theta_{3}$ axis. This translation is not a linear translation in real space, but rather it is a rotation about an axis. Translation along $\theta_{2}$ does not occur.

In Table 3, the number of equivalent positions is greater in most cases than the number of equivalent

\section{Table 2. Numbering of the rotation-function space groups}

The Laue group of the rotated Patterson map $P_{1}$ is chosen from the left column and the Laue group of $P_{2}$ is chosen from the upper row. 
Table 3. Rotation-function space groups

\begin{tabular}{|c|c|c|c|c|c|}
\hline $\begin{array}{l}\text { No. of } \\
\text { the } \\
\text { rotation } \\
\text { space } \\
\text { group }\end{array}$ & $\begin{array}{l}\text { No. of } \\
\text { equivalent } \\
\text { positions }\end{array}$ & Symbol $^{b}$ & $\begin{array}{l}\text { Translation } \\
\text { along the } \\
\theta_{1} \text { axis }^{c}\end{array}$ & $\begin{array}{c}\text { Translation } \\
\text { along the } \\
\theta_{3} \text { axis }^{c}\end{array}$ & $\begin{array}{c}\text { Range of } \\
\text { the } \\
\text { asymmetric } \\
\text { unit }^{d}\end{array}$ \\
\hline 1 & 2 & $P n$ & $2 \pi$ & $2 \pi$ & $\begin{array}{l}0 \leq \theta_{1}<2 \pi \\
0 \leq \theta_{2} \leq \pi \\
0 \leq \theta_{3}<2 \pi\end{array}$ \\
\hline 2 & 4 & $P b n 2_{1}$ & $2 \pi$ & $2 \pi$ & $\begin{array}{l}0 \leq \theta_{1}<2 \pi \\
0 \leq \theta_{2} \leq \pi / 2 \\
0 \leq \theta_{3}<2 \pi\end{array}$ \\
\hline 3 & 4 & $P c$ & $\pi$ & $2 \pi$ & $\begin{array}{l}0 \leq \theta_{1}<\pi \\
0 \leq \theta_{2} \leq \pi \\
0 \leq \theta_{3}<2 \pi\end{array}$ \\
\hline 4 & 8 & $P b c 2$, & $\pi$ & $2 \pi$ & $\begin{array}{l}0 \leq \theta_{1}<\pi \\
0 \leq \theta_{2} \leq \pi / 2 \\
0 \leq \theta_{3}<2 \pi\end{array}$ \\
\hline 5 & 8 & $P c$ & $\pi / 2$ & $2 \pi$ & $\begin{array}{l}0 \leq \theta_{1}<\pi / 2 \\
0 \leq \theta_{2} \leq \pi \\
0 \leq \theta_{3}<2 \pi\end{array}$ \\
\hline 6 & 16 & $P b c 2_{1}$ & $\pi / 2$ & $2 \pi$ & $\begin{array}{l}0 \leq \theta_{1}<\pi / 2 \\
0 \leq \theta_{2} \leq \pi / 2 \\
0 \leq \theta_{3}<2 \pi\end{array}$ \\
\hline 7 & 6 & $P n$ & $2 \pi / 3$ & $2 \pi$ & $\begin{array}{l}0 \leq \theta_{1}<2 \pi / 3 \\
0 \leq \theta_{2} \leq \pi \\
0 \leq \theta_{3}<2 \pi\end{array}$ \\
\hline 8 & 12 & $P b n 2_{1}$ & $2 \pi / 3$ & $2 \pi$ & $\begin{array}{l}0 \leq \theta_{1}<2 \pi / 3 \\
0 \leq \theta_{2} \leq \pi / 2 \\
0 \leq \theta_{3}<2 \pi\end{array}$ \\
\hline 9 & 12 & $P c$ & $\pi / 3$ & $2 \pi$ & $\begin{array}{l}0 \leq \theta_{1}<\pi / 3 \\
0 \leq \theta_{2} \leq \pi \\
0 \leq \theta_{3}<2 \pi\end{array}$ \\
\hline 10 & 24 & $P b c 2_{1}$ & $\pi / 3$ & $2 \pi$ & $\begin{array}{l}0 \leq \theta_{1}<\pi / 3 \\
0 \leq \theta_{2} \leq \pi / 2 \\
0 \leq \theta_{3}<2 \pi\end{array}$ \\
\hline 11 & 4 & $P 2, n b$ & $2 \pi$ & $2 \pi$ & $\begin{array}{l}0 \leq \theta_{1}<2 \pi \\
0 \leq \theta_{2} \leq \pi / 2 \\
0 \leq \theta_{3}<2 \pi\end{array}$ \\
\hline 12 & 8 & Pbnb & $2 \pi$ & $2 \pi$ & $\begin{array}{l}0 \leq \theta_{1} \leq \pi / 2 \\
0 \leq \theta_{2}<\pi \\
0 \leq \theta_{3}<2 \pi\end{array}$ \\
\hline 13 & 8 & $P 2 c b$ & $\pi$ & $2 \pi$ & $\begin{array}{l}0 \leq \theta_{1}<\pi \\
0 \leq \theta_{2} \leq \pi / 2 \\
0 \leq \theta_{3}<2 \pi\end{array}$ \\
\hline 14 & 16 & $P b c b$ & $\pi$ & $2 \pi$ & $\begin{array}{l}0 \leq \theta_{1} \leq \pi / 2 \\
0 \leq \theta_{2} \leq \pi / 2 \\
0 \leq \theta_{3}<2 \pi\end{array}$ \\
\hline 15 & 16 & $P 2 c b$ & $\pi / 2$ & $2 \pi$ & $\begin{array}{l}0 \leq \theta_{1}<\pi / 2 \\
0 \leq \theta_{2} \leq \pi / 2 \\
0 \leq \theta_{3}<2 \pi\end{array}$ \\
\hline 16 & 32 & $P b c b$ & $\pi / 2$ & $2 \pi$ & $\begin{array}{l}0 \leq \theta_{1}<\pi / 2 \\
0 \leq \theta_{2}<\pi \\
0 \leq \theta_{3} \leq \pi / 2\end{array}$ \\
\hline 17 & 12 & $P 2, n b$ & $2 \pi / 3$ & $2 \pi$ & $\begin{array}{l}0 \leq \theta_{1}<2 \pi / 3 \\
0 \leq \theta_{2} \leq \pi / 2 \\
0 \leq \theta_{3}<2 \pi\end{array}$ \\
\hline 18 & 24 & Pbnb & $2 \pi / 3$ & $2 \pi$ & $\begin{array}{l}0 \leq \theta_{1}<2 \pi / 3 \\
0 \leq \theta_{2}<\pi \\
0 \leq \theta_{3} \leq \pi / 2\end{array}$ \\
\hline 19 & 24 & $P 2 c b$ & $\pi / 3$ & $2 \pi$ & $\begin{array}{l}0 \leq \theta_{1}<\pi / 3 \\
0 \leq \theta_{2} \leq \pi / 2 \\
0 \leq \theta_{3}<2 \pi\end{array}$ \\
\hline 20 & 48 & $P b c b$ & $\pi / 3$ & $2 \pi$ & $\begin{array}{l}0 \leq \theta_{1}<\pi / 3 \\
0 \leq \theta_{2}<\pi \\
0 \leq \theta_{3} \leq \pi / 2\end{array}$ \\
\hline 21 & 4 & $P a$ & $2 \pi$ & $\pi$ & $\begin{array}{l}0 \leq \theta_{1}<2 \pi \\
0 \leq \theta_{2} \leq \pi \\
0 \leq \theta_{3}<\pi\end{array}$ \\
\hline 22 & 8 & Pba2 & $2 \pi$ & $\pi$ & $\begin{array}{l}0 \leq \theta_{1}<2 \pi \\
0 \leq \theta_{2} \leq \pi / 2 \\
0 \leq \theta_{3}<\pi\end{array}$ \\
\hline 23 & 8 & $P m$ & $\pi$ & $\pi$ & $\begin{array}{l}0 \leq \theta_{1}<\pi \\
0 \leq \theta_{2} \leq \pi \\
0 \leq \theta_{3}<\pi\end{array}$ \\
\hline 24 & 16 & Pbm2 & $\pi$ & $\pi$ & $\begin{array}{l}0 \leq \theta_{1}<\pi \\
0 \leq \theta_{2} \leq \pi / 2 \\
0 \leq \theta_{3}<\pi\end{array}$ \\
\hline 25 & 16 & $P m$ & $\pi / 2$ & $\pi$ & $\begin{array}{l}0 \leq \theta_{1}<\pi / 2 \\
0 \leq \theta_{2} \leq \pi \\
0 \leq \theta_{3}<\pi\end{array}$ \\
\hline 26 & 32 & $P b m 2$ & $\pi / 2$ & $\pi$ & $\begin{array}{l}0 \leq \theta_{1}<\pi / 2 \\
0 \leq \theta_{2} \leq \pi / 2 \\
0 \leq \theta_{3}<\pi\end{array}$ \\
\hline
\end{tabular}

Table 3 (cont.)

\begin{tabular}{|c|c|c|c|c|}
\hline $\begin{array}{l}\text { No. of } \\
\text { the } \\
\text { rotation } \\
\text { space } \\
\text { group }\end{array}$ & $\begin{array}{l}\text { No. of } \\
\text { equivalent } \\
\text { positions }{ }^{a}\end{array}$ & Symbol $^{b}$ & $\begin{array}{c}\text { Translation } \\
\text { along the } \\
\theta_{1} \text { axis }^{c}\end{array}$ & $\begin{array}{c}\text { Translation } \\
\text { along the } \\
\theta_{3} \text { axis }^{c}\end{array}$ \\
\hline 27 & 12 & $P a$ & $2 \pi / 3$ & $\pi$ \\
\hline 28 & 24 & $P b a 2$ & $2 \pi / 3$ & $\pi$ \\
\hline 29 & 24 & $P m$ & $\pi / 3$ & $\pi$ \\
\hline
\end{tabular}

Range of
the
asymmetric unit $^{d}$

$0 \leq \theta_{1}<2 \pi / 3$ $0 \leq \theta_{2} \leq \pi$ $0 \leq \theta_{3}<\pi$ $0 \leq \theta_{1}<2 \pi / 3$ $0 \leq \theta_{2} \leq \pi / 2$ $0<\theta_{3}<\pi$ $0 \leq \theta_{1}<\pi / 3$ $0 \leq \theta_{2} \leq \pi$ $0 \leq \theta_{1}<\pi / 3$ $0 \leq \theta_{2} \leq \pi / 2$ $0<\theta_{3}<\pi$ $0 \leq \theta_{1}<2 \pi$ $0 \leq \theta_{2} \leq \pi / 2$ $0 \leq \theta_{3}<\pi$ $0 \leq \theta_{1} \leq \pi / 2$ $0 \leq \theta_{2}<\pi$ $0 \leq \theta_{3}<\pi$ $0 \leq \theta_{1}<\pi$ $0 \leq \theta_{2} \leq \pi / 2$ $0 \leq \theta_{3}<\pi$ $0 \leq \theta_{1} \leq \pi / 2$ $0 \leq \theta_{2} \leq \pi / 2$ $0 \leq \theta_{3}<\pi$ $0 \leq \theta_{1}<\pi / 2$ $0 \leq \theta_{2} \leq \pi / 2$ $0 \leq \theta_{3}<\pi$ $0 \leqslant \theta_{1}<\pi / 2$ $0 \leq \theta_{3} \leq \pi / 2$ $0 \leq \theta_{1}<2 \pi / 3$ $0 \leq \theta_{2} \leq \pi / 2$ $0 \leq \theta_{3}<\pi$

$0 \leq \theta_{1}<2 \pi / 3$

$0 \leq \theta_{2} \leq \pi / 2$ $0 \leq \theta_{3} \leq \pi / 2$ $0 \leq \theta_{1}<\pi / 3$ $0 \leq \theta_{2} \leq \pi / 2$ $0 \leq \theta_{3}<\pi$ $0 \leq \theta_{1}<\pi / 3$
$0 \leq \theta_{2} \leq \pi / 2$ $0 \leq \theta_{3} \leq \pi / 2$ $0 \leq \theta_{1}<2 \pi$ $0 \leq \theta_{2} \leq \pi$

$0 \leq \theta_{3}<\pi / 2$ $0 \leq \theta_{1}<2 \pi$ $0 \leq \theta_{2} \leq \pi / 2$ $0 \leq \theta_{3}<\pi / 2$

$0 \leq \theta_{1}<\pi$

$0 \leq \theta_{2} \leq \pi$

$0 \leq \theta_{3}<\pi / 2$

$0 \leq \theta_{1}<\pi$

$0 \leq \theta_{3}<\pi / 2$

$0 \leq \theta_{1}<\pi / 2$

$0 \leq \theta_{2} \leq \pi$

$0 \leq \theta_{3}<\pi / 2$

$0 \leq \theta_{1}<\pi / 2$

$0 \leq \theta_{2} \leq \pi / 2$

$0 \leq \theta_{3}<\pi / 2$

$0 \leq \theta_{1}<2 \pi / 3$

$0 \leq \theta_{2} \leq \pi$

$0 \leq \theta_{3}<\pi / 2$

$0 \leq \theta_{1}<2 \pi / 3$

$0 \leq \theta_{2} \leq \pi / 2$

$0 \leq \theta_{3}<\pi / 2$

$0 \leq \theta_{1}<\pi / 3$

$0 \leq \theta_{2} \leq \pi$

$0 \leq \theta_{3}<\pi / 2$

$0 \leq \theta_{1}<\pi / 3$

$0 \leq \theta_{2} \leq \pi / 2$

$0 \leq \theta_{3}<\pi / 2$

$0 \leq \theta_{1}<2 \pi$

$0 \leq \theta_{2} \leq \pi / 2$

$0 \leq \theta_{3}<\pi / 2$

$0 \leq \theta_{1}<2 \pi$

$0 \leq \theta_{2} \leq \pi / 2$
$0 \leq \theta_{3} \leq \pi / 4$ 
Table 3 (cont.)

Table 3 (cont.)

\begin{tabular}{|c|c|c|c|c|c|}
\hline $\begin{array}{l}\text { No. of } \\
\text { the } \\
\text { rotation } \\
\text { space } \\
\text { group }\end{array}$ & $\begin{array}{l}\text { No. of } \\
\text { equivalent } \\
\text { positions }{ }^{a}\end{array}$ & Symbol $^{h}$ & $\begin{array}{c}\text { Translation } \\
\text { along the } \\
\theta_{1} \text { axis }^{\mathrm{c}}\end{array}$ & $\begin{array}{c}\text { Translation } \\
\text { along the } \\
\theta_{3} \text { axis }^{c}\end{array}$ & $\begin{array}{c}\text { Range of } \\
\text { the } \\
\text { asymmetric } \\
\text { unit }^{d}\end{array}$ \\
\hline 53 & 32 & $P 2 m b$ & $\pi$ & $\pi / 2$ & $\begin{array}{l}0 \leq \theta_{1}<\pi \\
0 \leq \theta_{2} \leq \pi / 2 \\
0<\theta_{3}<\pi / 2\end{array}$ \\
\hline 54 & 64 & $P b m b$ & $\pi$ & $\pi / 2$ & $\begin{array}{l}0<\theta_{1}<\pi / 2 \\
0 \leq \theta_{2} \leq \pi / 2 \\
0 \leq \theta_{3}<\pi / 2\end{array}$ \\
\hline 55 & 64 & $P 2 m b$ & $\pi / 2$ & $\pi / 2$ & $\begin{array}{l}0 \leq \theta_{1}<\pi / 2 \\
0 \leq \theta_{2} \leq \pi / 2 \\
0 \leq \theta_{3}<\pi / 2\end{array}$ \\
\hline 56 & 128 & $P b m b$ & $\pi / 2$ & $\pi / 2$ & $\begin{array}{l}0 \leq \theta_{1} \leq \pi / 4 \\
0 \leq \theta_{2} \leq \pi / 2 \\
0 \leq \theta_{3}<\pi / 2\end{array}$ \\
\hline 57 & 48 & $P 2, a b$ & $2 \pi / 3$ & $\pi / 2$ & $\begin{array}{l}0 \leq \theta_{1}<2 \pi / 3 \\
0 \leq \theta_{2} \leq \pi / 2 \\
0 \leq \theta_{3}<\pi / 2\end{array}$ \\
\hline 58 & 96 & $P b a b$ & $2 \pi / 3$ & $\pi / 2$ & $\begin{array}{l}0 \leq \theta_{1}<2 \pi / 3 \\
0 \leq \theta_{2} \leq \pi / 2 \\
0<\theta_{3}<\pi / 4\end{array}$ \\
\hline 59 & 96 & $P 2 m b$ & $\pi / 3$ & $\pi / 2$ & $\begin{array}{l}0 \leq \theta_{1}<\pi / 3 \\
0 \leq \theta_{2} \leq \pi / 2 \\
0 \leq \theta_{3}<\pi / 2\end{array}$ \\
\hline 60 & 192 & $P b m b$ & $\pi / 3$ & $\pi / 2$ & $\begin{array}{l}0 \leq \theta_{1} \leq \pi / 6 \\
0 \leq \theta_{2} \leq \pi / 2 \\
0 \leq \theta_{3}<\pi / 2\end{array}$ \\
\hline 61 & 6 & $P n$ & $2 \pi$ & $2 \pi / 3$ & $\begin{array}{l}0 \leq \theta_{1}<2 \pi \\
0 \leq \theta_{2} \leq \pi \\
0 \leq \theta_{3}<2 \pi / 3\end{array}$ \\
\hline 62 & 12 & $P b n 2_{1}$ & $2 \pi$ & $2 \pi / 3$ & $\begin{array}{l}0 \leq \theta_{1}<2 \pi \\
0 \leq \theta_{2} \leq \pi / 2 \\
0<\theta_{3}<2 \pi / 3\end{array}$ \\
\hline 63 & 12 & $P_{C}$ & $\pi$ & $2 \pi / 3$ & $\begin{array}{l}0 \leq \theta_{1}<\pi \\
0 \leq \theta_{2}<\pi \\
0 \leq \theta_{3}<2 \pi / 3\end{array}$ \\
\hline 64 & 24 & $P b c 2_{1}$ & $\pi$ & $2 \pi / 3$ & $\begin{array}{l}0<\theta_{1}<\pi \\
0<\theta_{2} \leq \pi / 2 \\
0 \leq \theta_{3}<2 \pi / 3\end{array}$ \\
\hline 65 & 24 & $P c$ & $\pi / 2$ & $2 \pi / 3$ & $\begin{array}{l}0 \leq \theta_{1}<\pi / 2 \\
0 \leq \theta_{2} \leq \pi \\
0 \leq \theta_{3}<2 \pi / 3\end{array}$ \\
\hline 66 & 48 & $P b c 2_{1}$ & $\pi / 2$ & $2 \pi / 3$ & $\begin{array}{l}0 \leq \theta_{1}<\pi / 2 \\
0 \leq \theta_{2} \leq \pi / 2 \\
0 \leq \theta_{3}<2 \pi / 3\end{array}$ \\
\hline 67 & 18 & $P_{n}$ & $2 \pi / 3$ & $2 \pi / 3$ & $\begin{array}{l}0 \leq \theta_{1}<2 \pi / 3 \\
0 \leq \theta_{2} \leq \pi \\
0 \leq \theta_{3}<2 \pi / 3\end{array}$ \\
\hline 68 & 36 & Pbn2, & $2 \pi / 3$ & $2 \pi / 3$ & $\begin{array}{l}0 \leq \theta_{1}<2 \pi / 3 \\
0 \leq \theta_{2} \leq \pi / 2 \\
0 \leq \theta_{3}<2 \pi / 3\end{array}$ \\
\hline 69 & 36 & $P_{C}$ & $\pi / 3$ & $2 \pi / 3$ & $\begin{array}{l}0 \leq \theta_{1}<\pi / 3 \\
0 \leq \theta_{2} \leq \pi \\
0 \leq \theta_{3}<2 \pi / 3\end{array}$ \\
\hline 70 & 72 & $P b c 2$ & $\pi / 3$ & $2 \pi / 3$ & $\begin{array}{l}0 \leq \theta_{1}<\pi / 3 \\
0 \leq \theta_{2} \leq \pi / 2 \\
0 \leq \theta_{3}<2 \pi / 3\end{array}$ \\
\hline 71 & 12 & $P 2, n b$ & $2 \pi$ & $2 \pi / 3$ & $\begin{array}{l}0 \leq \theta_{1}<2 \pi \\
0 \leq \theta_{2} \leq \pi / 2 \\
0 \leq \theta_{3}<2 \pi / 3\end{array}$ \\
\hline 72 & 24 & Pbnb & $2 \pi$ & $2 \pi / 3$ & $\begin{array}{l}0 \leq \theta_{1} \leq \pi / 2 \\
0 \leq \theta_{2}<\pi \\
0 \leq \theta_{3}<2 \pi / 3\end{array}$ \\
\hline 73 & 24 & $P 2 c b$ & $\pi$ & $2 \pi / 3$ & $\begin{array}{l}0 \leq \theta_{1}<\pi \\
0 \leq \theta_{2} \leq \pi / 2 \\
0 \leq \theta_{3}<2 \pi / 3\end{array}$ \\
\hline 74 & 48 & $P b c b$ & $\pi$ & $2 \pi / 3$ & $\begin{array}{l}0 \leq \theta_{1} \leq \pi / 2 \\
0 \leq \theta_{2} \leq \pi / 2 \\
0 \leq \theta_{3}<2 \pi / 3\end{array}$ \\
\hline 75 & 48 & $P 2 c b$ & $\pi / 2$ & $2 \pi / 3$ & $\begin{array}{l}0 \leq \theta_{1}<\pi / 2 \\
0 \leq \theta_{2} \leq \pi / 2 \\
0 \leq \theta_{3}<2 \pi / 3\end{array}$ \\
\hline 76 & 96 & $P b c b$ & $\pi / 2$ & $2 \pi / 3$ & $\begin{array}{l}0 \leq \theta_{1} \leq \pi / 4 \\
0 \leq \theta_{2} \leq \pi / 2 \\
0 \leq \theta_{3}<2 \pi / 3\end{array}$ \\
\hline 77 & 36 & $P 2, n b$ & $2 \pi / 3$ & $2 \pi / 3$ & $\begin{array}{l}0 \leq \theta_{1}<2 \pi / 3 \\
0 \leq \theta_{2} \leq \pi / 2 \\
0 \leq \theta_{3}<2 \pi / 3\end{array}$ \\
\hline
\end{tabular}

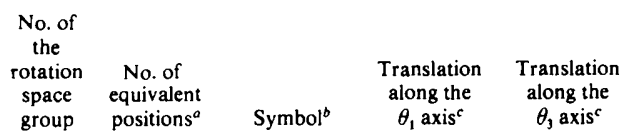

Range of

the asymmetric unit $^{d}$$$
78
$$

78

Pbnb

$2 \pi / 3$

79

$P 2 c b$

$\pi / 3$

$2 \pi / 3$

$0 \leq \theta_{1} \leq \pi / 6$

$0 \leq \theta_{2} \leq \pi$

$0 \leq \theta_{3}<2 \pi / 3$

$0<\theta_{1}<\pi / 3$

$0 \leq \theta_{2} \leq \pi / 2$

$0 \leq \theta_{3}<2 \pi / 3$

80

$P b c b$

$\pi / 3$

$2 \pi / 3$

$0<\theta_{2}<\pi / 2$

$0 \leq \theta_{3}<2 \pi / 3$

81

12

$P a$

$2 \pi$

$\pi / 3$

82

Pba2

$2 \pi$

$\pi / 3$

$0 \leq \theta_{1}<2 \pi$

$0 \leq \theta_{2} \leq \pi$

$0 \leq \theta_{1}<2 \pi$

$0 \leq \theta_{2}<\pi / 2$

$0 \leq \theta_{3}<\pi / 3$

83

Pm

84

Pbm2

$\pi / 3$

$0 \leq \theta_{1}<\pi$

$0 \leq \theta_{2} \leq \pi$

$0 \leq \theta_{3}<\pi / 3$

$0 \leq \theta_{1}<\pi$

$0 \leq \theta_{2} \leq \pi / 2$

$0 \leq \theta_{3}<\pi / 3$

$0 \leq \theta_{1}<\pi / 2$

$0 \leq \theta_{2} \leq \pi$

$0 \leq \theta_{3}<\pi / 3$

$0 \leq \theta_{1}<\pi / 2$

$0 \leq \theta_{2} \leq \pi / 2$

$0 \leq \theta_{3}<\pi / 3$

$0 \leq \theta_{1}<2 \pi / 3$

$0 \leq \theta_{2} \leq \pi$

$0 \leq \theta_{3}<\pi / 3$

$87 \quad 36$

$P a$

$2 \pi / 3$

$\pi / 3$

88

$\mathrm{Pba} 2$

$2 \pi / 3$

$\pi / 3$

$89 \quad 72$

Pm

$\pi / 3$

$\pi / 3$

90

144

Pbm2

$\pi / 3$

$\pi / 3$

$0 \leq \theta_{1}<2 \pi / 3$

$0 \leq \theta_{3}<\pi / 3$

$0 \leq \theta_{3}<\pi / 3$
$0 \leq \theta_{1}<\pi / 3$

$0 \leq \theta_{2} \leq \pi$

$0 \leq \theta_{3}<\pi / 3$

$0 \leq \theta_{1}<\pi / 3$

$0 \leq \theta_{2} \leq \pi / 2$

$0 \leq \theta_{3}<\pi / 3$

$0 \leq \theta_{1}<2 \pi$

$0 \leq \theta_{2} \leq \pi / 2$

$0 \leq \theta_{3}<\pi / 3$

$\begin{array}{lllll}91 & 24 & P 2, a b & 2 \pi & \pi / 3\end{array}$

92

Pbab

$2 \pi$

$\pi / 3$

$0 \leq \theta_{1} \leq \pi / 2$

$0 \leq \theta_{2}<\pi$

$0 \leq \theta_{3}<\pi / 3$

$93 \quad 48$

$P 2 m b$

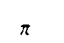

$\pi / 3$

$0 \leq \theta_{1}<\pi$

$0 \leq \theta_{2} \leq \pi / 2$

$0 \leq \theta_{1} \leq \pi / 2$

$0 \leq \theta_{1} \leq \pi / 2$
$0 \leq \theta_{2} \leq \pi / 2$

$0 \leq \theta_{3} \leq \pi / 2$

$0 \leq \theta_{1}<\pi / 2$

$0 \leq \theta_{2} \leq \pi / 2$

$0 \leq \theta_{3}<\pi / 3$

$0 \leq \theta_{1} \leq \pi / 4$

$0 \leq \theta_{2} \leq \pi / 2$

$0 \leq \theta_{3}<\pi / 3$

$0 \leq \theta_{1}<2 \pi / 3$

$0 \leq \theta_{2} \leq \pi / 2$

$0 \leq \theta_{3}<\pi / 3$

$0 \leq \theta_{1}<2 \pi / 3$

$0 \leq \theta_{2} \leq \pi / 2$

$0 \leq \theta_{3} \leq \pi / 6$

$0 \leq \theta_{1}<\pi / 3$

$0 \leq \theta_{2} \leq \pi / 2$

$0 \leq \theta_{3}<\pi / 3$

$0 \leq \theta_{1} \leq \pi / 6$

$0 \leq \theta_{2} \leq \pi / 2$

$\begin{array}{llll}100 & 288 \quad \pi / 3 & \pi / 3 & 0 \leq \theta_{1} \leq \pi / 6 \\ & & 0 \leq \theta_{2} \leq \pi / 2 \\ & & 0 \leq \theta_{3}<\pi / 3\end{array}$

${ }^{a}$ This is the number of equivalent positions in the rotation unit cell.

- Each symbol retains the order $\theta_{1}, \theta_{2}, \theta_{3}$. The monoclinic space groups have the $b$-axis unique setting.

c This is a translation symmetry: e.g. for the case of $\pi / 2$ translation along the $\theta_{1}$ axis, $\theta_{1}, \theta_{2}, \theta_{3}$ goes to $\pi / 2+\theta_{1}, \theta_{2}, \theta_{3}$ and $\pi+\theta_{1}, \theta_{2}, \theta_{3}$ and $3 \pi / 2+\theta_{1}, \theta_{2}, \theta_{3}$. All other equivalent positions in the basic rotation space group are similarly translated.

${ }^{d}$ Several consistent sets of ranges exist but the one with the minimum range of $\theta_{2}$ is listed. 
Table 4. The 16 basic rotation-function space groups and their equivalent positions

\begin{tabular}{|c|c|c|c|c|c|c|}
\hline $\mathrm{Pa}$ & $\theta_{1}$, & $\theta_{2}$, & $\theta_{3}$ & $\pi+\theta_{1}$ & $-\theta_{2}$, & $\theta_{3}$ \\
\hline$P c$ & $\theta_{1}$, & $\theta_{2}$, & $\theta_{3}$ & $\theta_{1}$ & $-\theta_{2}$, & $\pi+\theta_{3}$ \\
\hline$P m$ & $\theta_{1}$, & $\theta_{2}$, & $\theta_{3}$ & $\theta_{1}$, & $-\theta_{2}$, & $\theta_{3}$ \\
\hline$P n$ & $\theta_{1}$, & $\theta_{2}$ & $\theta_{3}$ & $\pi+\theta_{1}$ & $-\theta_{2}$, & $\pi+\theta_{3}$ \\
\hline $\mathrm{Pbm} 2$ & $\begin{array}{r}\theta_{1}, \\
-\theta_{1},\end{array}$ & $\begin{array}{r}\theta_{2}, \\
\pi+\theta_{2}\end{array}$ & $\begin{array}{l}\theta_{3} \\
\theta_{3}\end{array}$ & $\begin{array}{r}\theta_{1} \\
-\theta_{1}\end{array}$ & $\begin{array}{r}-\theta_{2} \\
\pi-\theta_{2}\end{array}$ & $\begin{array}{l}\theta_{3} \\
\theta_{3}\end{array}$ \\
\hline$P 2 m b$ & $\begin{array}{l}\theta_{1} \\
\theta_{1}\end{array}$ & $\begin{array}{r}\theta_{2} \\
-\theta_{2}\end{array}$ & $\begin{array}{l}\theta_{3} \\
\theta_{3}\end{array}$ & $\begin{array}{l}\theta_{1}, \\
\theta_{1},\end{array}$ & $\begin{array}{l}\pi+\theta_{2}, \\
\pi-\theta_{2},\end{array}$ & $\begin{array}{l}-\theta_{3} \\
-\theta_{3}\end{array}$ \\
\hline$P b c 2_{1}$ & $\begin{array}{r}\theta_{1} \\
-\theta_{1}\end{array}$ & $\begin{array}{r}\theta_{2}, \\
\pi+\theta_{2},\end{array}$ & $\begin{array}{l}\theta_{3} \\
\theta_{3}\end{array}$ & $\begin{array}{r}\theta_{1} \\
-\theta_{1},\end{array}$ & $\begin{array}{r}-\theta_{2}, \\
\pi-\theta_{2},\end{array}$ & $\begin{array}{l}\pi+\theta_{3} \\
\pi+\theta_{3}\end{array}$ \\
\hline$P 2, a b$ & $\begin{array}{r}\theta_{1}, \\
\pi+\theta_{1}\end{array}$ & $\begin{array}{r}\theta_{2} \\
-\theta_{2}\end{array}$ & $\begin{array}{l}\theta_{3} \\
\theta_{3}\end{array}$ & $\begin{array}{r}\theta_{1}, \\
\pi+\theta_{1},\end{array}$ & $\begin{array}{l}\pi+\theta_{2} \\
\pi-\theta_{2}\end{array}$ & $\begin{array}{l}-\theta_{3} \\
-\theta_{3}\end{array}$ \\
\hline$P b a 2$ & $\begin{array}{r}\theta_{1} \\
-\theta_{1}\end{array}$ & $\begin{array}{r}\theta_{2}, \\
\pi-\theta_{2},\end{array}$ & $\begin{array}{l}\theta_{3} \\
\theta_{3}\end{array}$ & $\begin{array}{l}\pi+\theta_{1}, \\
\pi-\theta_{1},\end{array}$ & $\begin{array}{r}-\theta_{2} \\
\pi+\theta_{2}\end{array}$ & $\begin{array}{c}\theta_{3} \\
\theta_{3}\end{array}$ \\
\hline$P 2 c b$ & $\begin{array}{l}\theta_{1} \\
\theta_{1},\end{array}$ & $\begin{array}{r}\theta_{2} \\
-\theta_{2}\end{array}$ & $\begin{array}{r}\theta_{3} \\
\pi+\theta_{3}\end{array}$ & $\begin{array}{l}\theta_{1} \\
\theta_{1}\end{array}$ & $\begin{array}{l}\pi+\theta_{2}, \\
\pi-\theta_{2},\end{array}$ & $\begin{array}{r}\pi-\theta_{3} \\
-\theta_{3}\end{array}$ \\
\hline$P b n 2_{1}$ & $\begin{array}{r}\theta_{1} \\
-\theta_{1}\end{array}$ & $\begin{array}{r}\theta_{2} \\
\pi-\theta_{2}\end{array}$ & $\begin{array}{r}\theta_{3} \\
\pi+\theta_{3}\end{array}$ & $\begin{array}{l}\pi+\theta_{1}, \\
\pi-\theta_{1},\end{array}$ & $\begin{array}{r}-\theta_{2}, \\
\pi+\theta_{2}\end{array}$ & $\begin{array}{r}\pi+\theta_{3} \\
\theta_{3}\end{array}$ \\
\hline$P 2, n b$ & $\begin{array}{r}\theta_{1}, \\
\pi+\theta_{1},\end{array}$ & $\begin{array}{r}\theta_{2} \\
-\theta_{2}\end{array}$ & $\begin{array}{r}\theta_{3} \\
\pi+\theta_{3}\end{array}$ & $\begin{array}{r}\theta_{1}, \\
\pi+\theta_{1},\end{array}$ & $\begin{array}{l}\pi+\theta_{2}, \\
\pi-\theta_{2},\end{array}$ & $\begin{array}{r}\pi-\theta_{3} \\
-\theta_{3}\end{array}$ \\
\hline$P b a b$ & $\begin{array}{r}\theta_{1}, \\
\pi+\theta_{1}, \\
-\theta_{1}, \\
\pi-\theta_{1},\end{array}$ & $\begin{array}{r}\theta_{2}, \\
-\theta_{2}, \\
-\theta_{2}, \\
\theta_{2},\end{array}$ & $\begin{array}{r}\theta_{3} \\
\theta_{3} \\
-\theta_{3} \\
-\theta_{3}\end{array}$ & $\begin{array}{r}\theta_{1}, \\
\pi+\theta_{1}, \\
-\theta_{1}, \\
\pi-\theta_{1},\end{array}$ & $\begin{array}{l}\pi+\theta_{2}, \\
\pi-\theta_{2}, \\
\pi-\theta_{2}, \\
\pi+\theta_{2},\end{array}$ & $\begin{array}{r}-\theta_{3} \\
-\theta_{3} \\
\theta_{3} \\
\theta_{3}\end{array}$ \\
\hline$P b m b$ & $\begin{array}{r}\theta_{1} \\
\theta_{1} \\
-\theta_{1} \\
-\theta_{1},\end{array}$ & $\begin{array}{r}\theta_{2} \\
-\theta_{2} \\
-\theta_{2} \\
\theta_{2}\end{array}$ & $\begin{array}{r}\theta_{3} \\
\theta_{3} \\
-\theta_{3} \\
-\theta_{3}\end{array}$ & $\begin{array}{r}\theta_{1}, \\
\theta_{1}, \\
-\theta_{1}, \\
-\theta_{1},\end{array}$ & $\begin{array}{l}\pi+\theta_{2}, \\
\pi-\theta_{2}, \\
\pi-\theta_{2}, \\
\pi+\theta_{2},\end{array}$ & $\begin{array}{r}-\theta_{3} \\
-\theta_{3} \\
\theta_{3} \\
\theta_{3}\end{array}$ \\
\hline$P b c b$ & $\begin{array}{r}\theta_{1}, \\
\theta_{1}, \\
-\theta_{1}, \\
-\theta_{1},\end{array}$ & $\begin{array}{r}\theta_{2}, \\
-\theta_{2}, \\
-\theta_{2}, \\
\theta_{2},\end{array}$ & $\begin{array}{r}\theta_{3} \\
\pi+\theta_{3} \\
-\theta_{3} \\
\pi-\theta_{3}\end{array}$ & $\begin{array}{r}\theta_{1}, \\
\theta_{1}, \\
-\theta_{1}, \\
-\theta_{1},\end{array}$ & $\begin{array}{l}\pi+\theta_{2}, \\
\pi-\theta_{2}, \\
\pi-\theta_{2}, \\
\pi+\theta_{2},\end{array}$ & $\begin{array}{r}\pi-\theta_{3} \\
-\theta_{3} \\
\pi+\theta_{3} \\
\theta_{3}\end{array}$ \\
\hline$P b n b$ & $\begin{array}{r}\theta_{1}, \\
\pi+\theta_{1}, \\
-\theta_{1}, \\
\pi-\theta_{1},\end{array}$ & $\begin{array}{r}\theta_{2} \\
-\theta_{2} \\
-\theta_{2} \\
\theta_{2}\end{array}$ & $\begin{array}{r}\theta_{3} \\
\pi+\theta_{3} \\
-\theta_{3} \\
\pi-\theta_{3}\end{array}$ & $\begin{array}{r}\theta_{1}, \\
\pi+\theta_{1}, \\
-\theta_{1}, \\
\pi-\theta_{1},\end{array}$ & $\begin{array}{l}\pi+\theta_{2}, \\
\pi-\theta_{2}, \\
\pi-\theta_{2}, \\
\pi+\theta_{2},\end{array}$ & $\begin{array}{r}\pi-\theta_{3} \\
-\theta_{3} \\
\pi+\theta_{3} \\
\theta_{3}\end{array}$ \\
\hline
\end{tabular}

positions dictated by the space-group symbol which is listed. This is due to the translation along the $\theta_{1}$ and/or the $\theta_{3}$ axis as indicated. In all instances where the basic rotation space group is $P a, P c, P m$ or $P n$ the asymmetric unit has $\theta_{2}$ limits $0 \leq \theta_{2} \leq \pi$. With a few exceptions (rotation space groups 12, 16, 18, 20, 32, 72 and 78), the other rotation space groups have $\theta_{2}$ limits $0 \leq \theta_{2} \leq \pi / 2$. In the seven exceptions listed, an upper limit of $\pi$ for $\theta_{2}$ was required in order for a continuous asymmetric unit to be chosen. If the basic rotationfunction space group is not $P b m b, P b a b, P b c b$ or $P b n b$ then the $\theta_{1}$ and $\theta_{3}$ limits on the asymmetric unit are equal to the allowed translations in $\theta_{1}$ and $\theta_{3}$. For these four basic rotation-function space groups of highest symmetry $\theta_{1}$ or $\theta_{3}$ limits less than the $\theta_{1}$ and $\theta_{3}$ translations are possible.

Crowther (1972) has used a definition of the rotation angles $(\alpha, \beta$ and $\gamma)$ in his fast rotation function program which is different from that adopted by Rossmann \& Blow (1962). However, a simple relationship exists between the two systems, viz: $\theta_{1}=\alpha+\pi / 2, \theta_{2}=\beta$ and $\theta_{3}=\gamma-\pi / 2$. Moreover, Crowther's program always computes ranges of $\alpha$ and $\gamma$ from 0 to $2 \pi / p$, where $p$ is the rotational symmetry along the axis about which the $\alpha$ rotation takes place. The symmetry of the Patterson map which is rotated affects the range of $\alpha$, and the symmetry of the stationary Patterson map affects the range of $\gamma$. This treatment is equivalent to acknowledging the translational symmetry which we observed in the equivalent positions of the rotation-function space groups. Therefore, the asymmetric units tabulated in Table 3 are appropriate for determining the range of $\beta$ required to cover the unique portion of rotation space. Crowther's program reindexes monoclinic data to the $c$-axis unique setting so those rotation space groups involving the $b$-axis unique setting will not occur. When the rotation space-group symmetry is $P b a b, P b m b, P b c b$ or $P b n b$ the asymmetric unit listed in Table 3 is smaller in $\theta_{1}$ or $\theta_{3}$ than is deduced from consideration of the translational symmetry only.

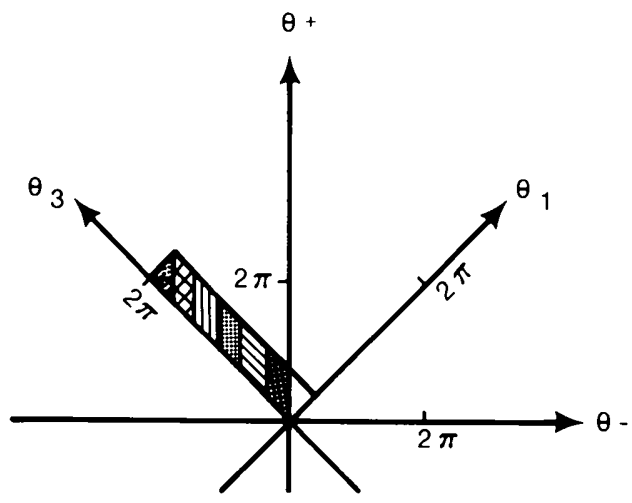

(a)

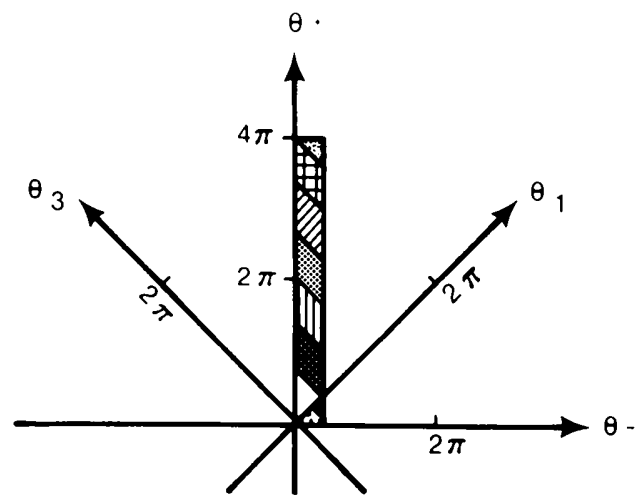

(b)

Fig. 1. Derivation of asymmetric unit in $\theta_{+}, \theta_{-}$space for rotation space group 19. $\theta_{1}$ translation is $\pi / 3, \theta_{3}$ translation is $2 \pi$, and $\theta_{+}$translation is $4 \pi$. Each segment of the asymmetric unit in $(a)$ is moved by allowed translations parallel to $\theta_{1}$ or $\theta_{3}$ to the similarly marked segment in $(b)$. This transfer does not involve $\theta_{2}$. Consequently, the Eulerian space asymmetric unit of $0 \leq \theta_{1} \leq$ $\pi / 3,0 \leq \theta_{2} \leq \pi / 2,0 \leq \theta_{3} \leq 2 \pi$ becomes $0 \leq \theta_{+}<4 \pi, 0 \leq \theta_{2} \leq$ $\pi / 2,0 \leq \theta_{-} \leq \pi / 3$ in $\theta_{+}, \theta_{-}$space. 
However, in all instances but one, the $\theta_{2}(\beta)$ range required will be that which is tabulated. The one exception is space group 78 where we chose a contiguous asymmetric unit $0 \leq \theta_{1} \leq \pi / 6,0 \leq \theta_{2} \leq \pi, 0$ $\leq \theta_{3}<2 \pi / 3$. A $\beta$ range from 0 to $\pi / 2$ will suffice for Crowther's treatment since the $\alpha$ and $\gamma$ will both range from 0 to $2 \pi / 3$. In the rotation space groups of symmetry $P b a b, P b m b, P b c b$ and $P b n b$ there will be more than one copy of the asymmetric unit in the output.

\section{Derivation of asymmetric units in $\theta_{+}, \theta_{-}$space}

Lattman (1972) showed that if the coordinates of the rotation function are $\theta_{+}, \theta_{2}$ and $\theta_{-}$where $\theta_{+}=\theta_{1}+\theta_{3}$ and $\theta_{-}=\theta_{1}-\theta_{3}$, then the coordinates are locally orthogonal and the sample points are associated with equal volumes so that more symmetrical peaks occur. He also presented a graphical means for determining the asymmetric unit in $\theta_{+}, \theta_{-}$space. Fig. 1 demon- strates the application of this method to rotation space group 19, which served as an example above. In this method, translations of portions of the asymmetric unit are allowed along the $\theta_{1}$ and $\theta_{3}$ axes as dictated by the rotation space group and listed in Table 3. Translations in the $\theta_{+}$direction sometimes are required. The translational equivalent in $\theta_{+}$is twice the translation which is a multiple of both the $\theta_{1}$ and the $\theta_{3}$ translations. For example, if the $\theta_{1}$ translation is $\pi / 3$ and the $\theta_{3}$ translation is $\pi / 2, \pi$ is a multiple of both of these so that a $2 \pi$ translation in $\theta_{+}$is allowed.

In order to derive the asymmetric units of all of the rotation space groups in $\theta_{+}, \theta_{-}$space, the rotationfunction space groups are best arranged according to translational symmetry in $\theta_{1}$ and $\theta_{3}$. Our results for the 100 rotation-function space groups are given in Table 5. In all instances but two (rotation space groups 58 and 76), the minimum asymmetric unit in $\theta_{+}, \theta_{-}$space may run in $\theta_{-}$from 0 to an upper limit which is equal to the translational symmetry in $\theta_{1}$ or $\theta_{3}$, whichever is smaller. In a majority of cases, a rectangular,

Table 5. Asymmetric units in $\theta_{+}, \theta_{-}$space

The rotation space groups are divided according to translational symmetry in $\theta_{1}$ (horizontal) and $\theta_{3}$ (vertical). Within each square, the first line lists the numbers of the four rotation space groups which have the indicated translational symmetry. If the list is followed by + the true asymmetric unit for all four space groups is smaller than the rectangular unit listed, while if the list is followed by * such is the case only for the last rotation space group listed. If only one set of $\theta_{+}, \theta_{-}$limits is listed it applies to all four space groups; when a second set of limits is listed it applies only to the fourth space group listed. The $\theta_{2}$ limits are the same as shown in Table 3 with two exceptions. Rotation space groups 12 and 78 , marked with $\S$, have limits $0 \leq \theta_{2} \leq \pi / 2$ in $\theta_{+}, \theta_{-}$space.

\begin{tabular}{|c|c|c|c|c|c|}
\hline & $\pi / 3$ & $\pi / 2$ & $2 \pi / 3$ & $\pi$ & $2 \pi$ \\
\hline & $89,90,99,100 *$ & $85,86,95,96+$ & $87,88,97,98 *$ & $83,84,93,94^{*}$ & $81,82,91,92 *$ \\
\hline \multirow[t]{2}{*}{$\pi / 3$} & $\begin{array}{l}0 \leq \theta_{+}<2 \pi / 3 \\
0 \leq \theta_{-} \leq \pi / 3\end{array}$ & $\begin{array}{l}-\pi / 3 \leq \theta_{+} \leq \pi \\
0 \leq \theta_{-} \leq \pi / 3 \\
-\pi / 3 \leq \theta_{+} \leq \pi / 2 \\
0 \leq \theta_{-} \leq \pi / 3\end{array}$ & $\begin{array}{l}0 \leq \theta_{+}<4 \pi / 3 \\
0 \leq \theta_{-} \leq \pi / 3\end{array}$ & $\begin{array}{l}0 \leq \theta_{+}<2 \pi \\
0 \leq \theta_{-} \leq \pi / 3 \\
-\pi / 3 \leq \theta_{+} \leq \pi \\
0 \leq \theta_{-} \leq \pi / 3\end{array}$ & $\begin{array}{l}0 \leq \theta_{+}<4 \pi \\
0 \leq \theta_{-} \leq \pi / 3 \\
-\pi / 3 \leq \theta_{+} \leq 3 \pi \\
0 \leq \theta_{-} \leq \pi / 3\end{array}$ \\
\hline & $49,50,59,60^{+}$ & $45,46,55,56 \star$ & $47,48,57,58^{\dagger}$ & $43,44,53,54 *$ & $41,42,51,52^{\circ}$ \\
\hline \multirow[t]{2}{*}{$\pi / 2$} & $\begin{array}{l}0 \leq \theta_{+} \leq 4 \pi / 3 \\
0 \leq \theta_{-} \leq \pi / 3 \\
0 \leq \theta_{+} \leq 5 \pi / 6 \\
0 \leq \theta_{-} \leq \pi / 3 \\
\end{array}$ & $\begin{array}{l}0 \leq \theta_{+}<\pi \\
0 \leq \theta_{-} \leq \pi / 2\end{array}$ & $\begin{array}{l}-\pi / 2 \leq \theta_{+} \leq 4 \pi / 3 \\
0 \leq \theta_{-} \leq \pi / 2 \\
0 \leq \theta_{+} \leq 7 \pi / 6 \\
0 \leq \theta_{-} \leq 2 \pi / 3\end{array}$ & $\begin{array}{l}0 \leq \theta_{+}<2 \pi \\
0 \leq \theta_{-} \leq \pi / 2 \\
-\pi / 2 \leq \theta_{+} \leq \pi / 2 \pi \\
0 \leq \theta_{-} \leq \pi / 2\end{array}$ & $\begin{array}{l}0 \leq \theta_{+}<4 \pi \\
0 \leq \theta_{-} \leq \pi / 2\end{array}$ \\
\hline & $69,70,79,80$ & $65,66,75,76^{\dagger}$ & $67,68,77,78=5$ & $63,64,73,74^{t}$ & $61,62,71,72$ \\
\hline \multirow[t]{2}{*}{$2 \pi / 3$} & $\begin{array}{l}0 \leq \theta_{+}<4 \pi / 3 \\
0 \leq \theta_{-} \leq \pi / 3\end{array}$ & $\begin{array}{l}0 \leq \theta_{+} \leq 11 \pi / 6 \\
0 \leq \theta_{-} \leq \pi / 2 \\
0 \leq \theta_{+} \leq 7 \pi / 6 \\
-\pi / 6 \leq \theta_{-} \leq \pi / 2\end{array}$ & $\begin{array}{l}0 \leq \theta_{+}<4 \pi / 3 \\
0 \leq \theta_{-} \leq 2 \pi / 3\end{array}$ & $\begin{array}{l}-2 \pi / 3 \leq \theta+\leq 2 \pi \\
0 \leq \theta-\leq 2 \pi / 3 \\
-2 \pi / 3 \leq \theta_{+} \leq \pi \\
0 \leq \theta-\leq 2 \pi / 3\end{array}$ & $\begin{array}{l}0 \leq \theta_{+}<4 \pi \\
0 \leq \theta_{-} \leq 2 \pi / 3 \\
-2 \pi / 3 \leq \theta_{+} \leq \pi \\
0 \leq \theta_{-} \leq 2 \pi / 3\end{array}$ \\
\hline & $29,30,39,40 *$ & $25,26,35,36$ * & $27,28,37,38^{+}$ & $23,24,33,34^{*}$ & $21,22,31,32 \star$ \\
\hline \multirow[t]{2}{*}{$\pi$} & $\begin{array}{l}0 \leq \theta_{+}<2 \pi \\
0 \leq \theta_{-} \leq \pi / 3 \\
0 \leq \theta_{+} \leq 4 \pi / 3 \\
0 \leq \theta_{-} \leq \pi / 3 \\
\end{array}$ & $\begin{array}{l}0 \leq \theta_{+}<2 \pi \\
0 \leq \theta_{-} \leq \pi / 2 \\
0 \leq \theta_{+} \leq 3 \pi / 2 \\
0 \leq \theta_{-}<\pi / 2\end{array}$ & $\begin{array}{l}0 \leq \theta_{+} \leq 8 \pi / 3 \\
0 \leq \theta_{-} \leq 2 \pi / 3 \\
0 \leq \theta_{+} \leq 5 \pi / 3 \\
0 \leq \theta_{-} \leq 2 \pi / 3\end{array}$ & $\begin{array}{l}0 \leq \theta_{+}<2 \pi \\
0 \leq \theta_{-} \leq \pi\end{array}$ & $\begin{array}{c}0 \leq \theta_{+}<4 \pi \\
0 \leq \theta_{-} \leq \pi \\
0 \leq \theta_{+} \leq \pi \\
-\pi \leq \theta_{-} \leq \pi\end{array}$ \\
\hline & $9,10,19,20 *$ & $5,6,15,16$ & $7,8,17,18$ & $3,4,13,14^{\star}$ & $1,2,11,12.5$ \\
\hline $2 \pi$ & $\begin{array}{l}0 \leq \theta_{+}<4 \pi \\
0 \leq \theta_{-} \leq \pi / 3 \\
0 \leq 0_{+} \leq 4 \pi / 3 \\
0 \leq \theta_{-} \leq \pi / 3\end{array}$ & $\begin{array}{l}0 \leq \theta_{+}<4 \pi \\
0 \leq \theta_{-} \leq \pi / 2 \\
0 \leq \theta_{+} \leq 3 \pi / 2 \\
0 \leq \theta_{-} \leq \pi / 2\end{array}$ & $\begin{array}{l}0 \leq \theta_{+}<4 \pi \\
0 \leq \theta_{-} \leq 2 \pi / 3 \\
0 \leq \theta_{+} \leq 5 \pi / 3 \\
0 \leq 0-\leq 2 \pi / 3\end{array}$ & $\begin{array}{l}0 \leq \theta_{+}<4 \pi \\
0 \leq \theta_{-} \leq \pi\end{array}$ & $\begin{array}{l}0 \leq \theta_{+}<4 \pi \\
0 \leq \theta_{-} \leq 2 \pi\end{array}$ \\
\hline
\end{tabular}


continuous asymmetric unit can be deduced in $\theta_{+}, \theta_{-}$ space. For these cases the limits in $\theta_{+}$are from 0 to twice the translational symmetry in $\theta_{1}$ or $\theta_{3}$, whichever is larger. In the other instances where the asymmetric unit is not rectangular or not continuous, the asymmetric unit listed in Table 5 will contain some redundancy. A space-group-specific rotation-function computer program which only calculates the unique portions of the asymmetric units listed in Table 5 is certainly feasible.

\section{Discussion}

The rotation function is now being applied widely to elucidate macromolecular structures. Rotation functions are calculated either in terms of Eulerian angles $\theta_{1}, \theta_{2}, \theta_{3}$ as described by Rossmann \& Blow (1962) or in the quasi-orthogonal angles $\theta_{+}, \theta_{2}$ and $\theta_{-}$. Sometimes, if an internal symmetry axis can be anticipated, the spherical polar angles $\varphi$ and $\psi$ and the azimuthal angle $\chi$ are used. However, the symmetry of the rotation function is more difficult to define in this system.

In several instances rotation-function space groups have been explicitly stated in the literature. These studies provide confirmation of our assignment of rotation-function space groups for space groups 12 (Rossmann \& Blow, 1962), 22 (Wishner, Ward, Lattman \& Love, 1975), 24 (Tollin, Main \& Rossmann, 1966), 31 (Lattman \& Love, 1970; Ward, Wishner, Lattman \& Love, 1975), 32 (Burnett \& Rossmann, 1971) and 34 (Rossmann, Ford, Watson \& Banaszak, 1972). Although many of these workers did not choose asymmetric-unit limits the same as those listed in Tables 4 and 5, their choices are equivalent to ours. In a study which uses rotation space group 61 , the space-group name is not given but the limits on $\theta_{+}$, $\theta_{2}$ and $\theta_{-}$which were used are consistent with our asymmetric unit (Schmidt, Herriott \& Lattman, 1974).

We wish to thank Dr Dick van der Helm for careful scrutiny of Jyh-Hwang Jih's PhD dissertation. Financial support was provided by grant no. PCM77-27337 from the National Science Foundation.

\section{References}

Burnett, R. M. \& Rossmann, M. G. (1971). Acta Cryst. B27, 1378-1387.

Crowther, R. A. (1972). The Molecular Replacement Method, edited by M. G. RossmanN, pp. 173-178. New York: Gordon and Breach.

International Tables for X-ray Crystallography (1969). Vol. I. Birmingham: Kynoch Press.

LatTMAN, E. E. (1972). Acta Cryst. B28, 1065-1068.

Lattman, E. E. \& Love, W. E. (1970). Acta Cryst. B26, 1854-1857.

Rossmann, M. G. \& Blow, D. M. (1962). Acta Cryst. 15, 24-31.

Rossmann, M. G., Ford, G. C., Watson, H. C. \& BANASZAK, L. J. (1972). J. Mol. Biol. 64, 237-249.

Schmidt, M. F., Herriotr, J. R. \& LatTMAN, E. E. (1974). J. Mol. Biol. 84, 97-101.

Tollin, P., Main, P. \& Rossmann, M. G. (1966). Acta Cryst. 20, 404-407.

W ARD, K. B., Wishner, B. C., Lattman, E. E. \& Love, W. E. (1975). J. Mol. Biol. 98, 161-177.

Wishner, B. C., Ward, K. B., LatTMan, E. E. \& Love, W. E. (1975). J. Mol. Biol. 98, 179-194.

Acta Cryst. (1980). A36, 884-888

\title{
Coloured Plane Groups
}

\author{
BY J. D. JARRATT \\ Pure Mathematics Department, University of Sydney, Sydney, Australia \\ AND R. L. E. SCHWARZENBERGER \\ Science Education Department, University of Warwick, Coventry, England
}

(Received 9 August 1979; accepted 4 June 1980)

\begin{abstract}
The 46 black and white plane groups are well known. The corresponding colour groups with more than two colours are extremely numerous. We give a listing of the 935 groups with $N$ colours for $N$ lying between 2 and 15 inclusive.
\end{abstract}

0567-7394/80/060884-05\$01.00

\section{Introduction}

Consider an $n$-dimensional space group $G$ whose elements permute $N$ colours transitively and let $G_{1}$ be the subgroup keeping the first colour fixed. Then the index of $G_{1}$ in $G$ is $N$ and the colours correspond naturally to the cosets. The effect of any member of $G$ 